THP-31-(T5)

\title{
Multiphoton identification of infectious fungi in dermatology
}

\author{
Ruei-Jhih Wu1, Sung-Jan Lin2, Chen-Yuan Dong1
}

1: Microscopic Biophysics Laboratory, Department of Physics, National Taiwan University, Taipei, Taiwan 106, ROC

2: Department of Dermatology, National Taiwan University Hospital and College of Medicine, Taipei, Taiwa 100, ROC

\begin{abstract}
*To whom correspondence should be addressed : cydong@phys.ntu.edu.tw Tel.: 886-2-3366-5155 ; Fax : 886-2-3366-5244
\end{abstract}

\begin{abstract}
This work focuses on using multiphoton microscopy for classifying and characterizing the infectious fungi in dermatology. We imaged and found that four fungi, M.canis T. menta Fusariun Asp.flavus, can be identified using multiphoton imaging and that their luminescence is located in blue and green part of the spectrum.
\end{abstract}

\section{Motivation}

Microscopic identification of infectious fungi may be of value in the diagnose and treatment of their infections in the skin. However, traditional imaging of fungi in the skin is difficult due to the lack of contrast of the skin layers and the fungi.

Therefore, we propose to use multiphoton microscopy to acquire microscopic features capable of identifying infectious fungi in the skin.

The principles of two-photon excitation, a form of multiphoton excitation, is illustrated in Fig. 1. By using femtosecond, near-infrared excitation sources to induce the simultaneous absorption of two photons, fluorescence molecules typically excited by UV or visible photons can be used for sample excitation.

\section{$\underline{\text { Result }}$}

We have obtained the multiphoton images of four types of fungi M.canis T.menta Fusariun, and Asp.flavus and their images along with bright field images are shown in Figs. 2-5.

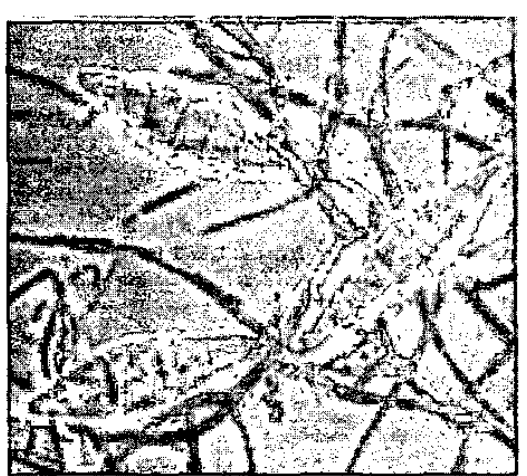

Figs. 1 M.canis on optic

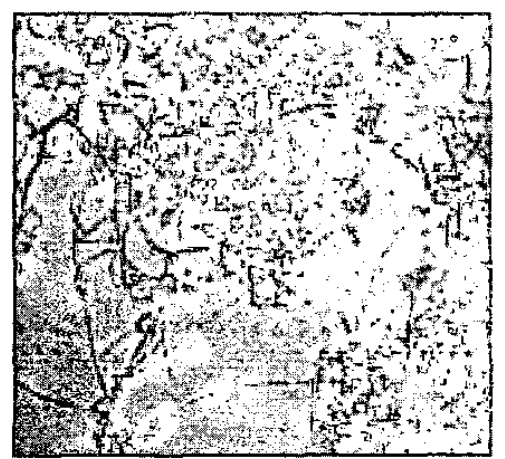

Fig. 4T.menta on optic

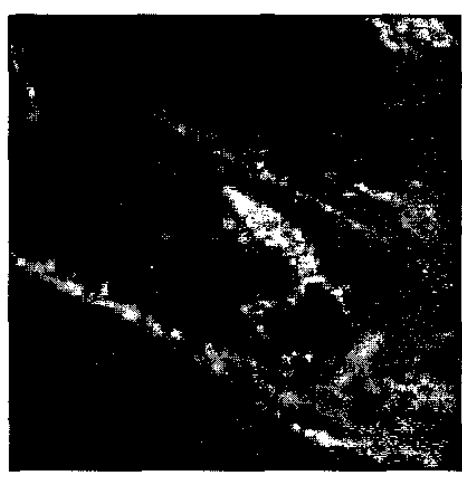

Figs. 2 M.canis on Two-Photon

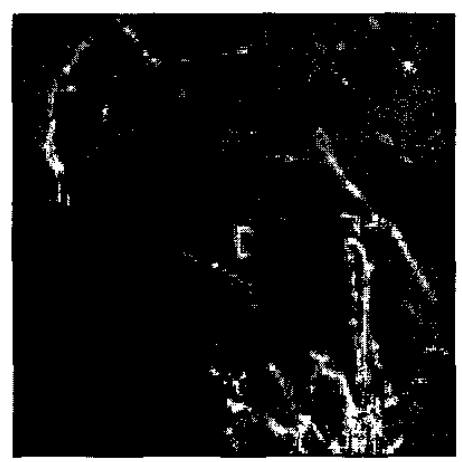

Fig. 5 T.menta on Two-Photon

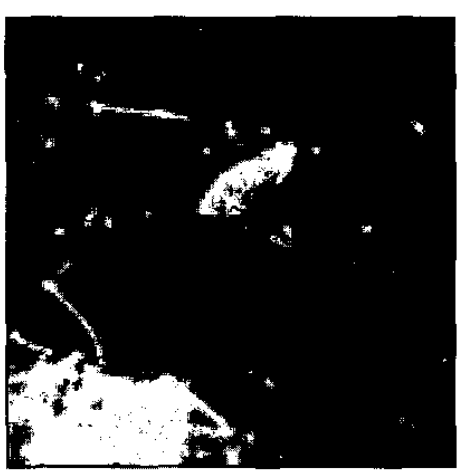

Figs. 3 M.canis on Two-Photon

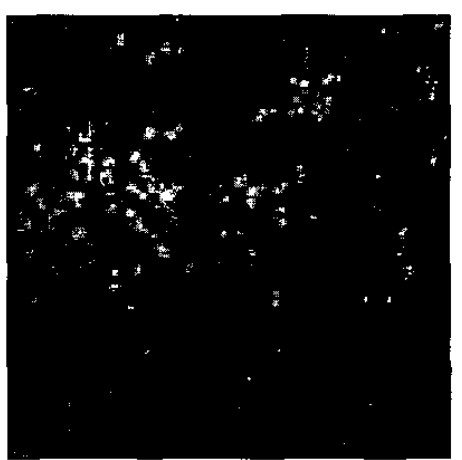

Fig. 6 T.menta on Two-Photon 
THP-31(T5)

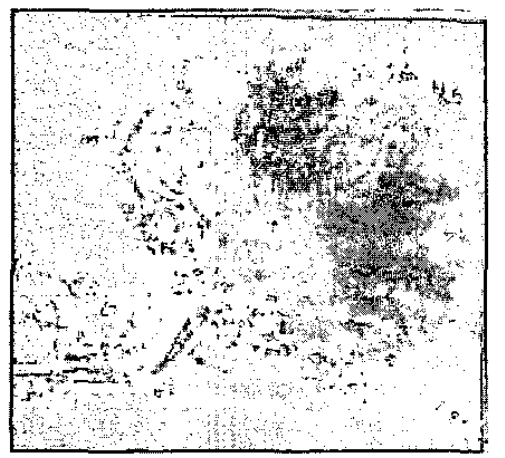

Fig. 7 Asp-flavus on opic

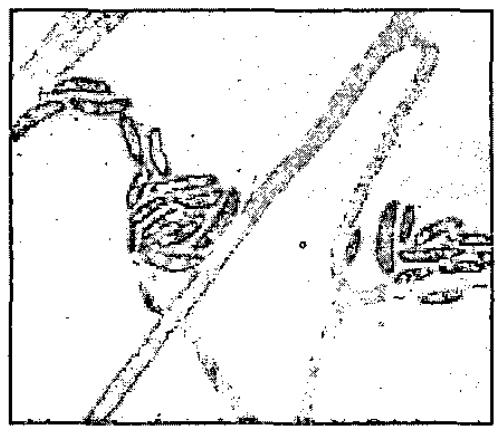

Fig. 10 Fusarium on optic

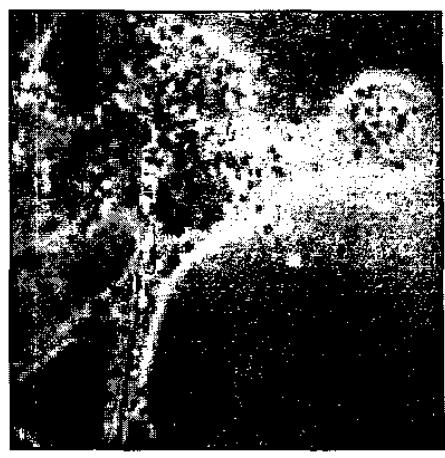

Fig. 8 Asp-flavus on Two-Photon

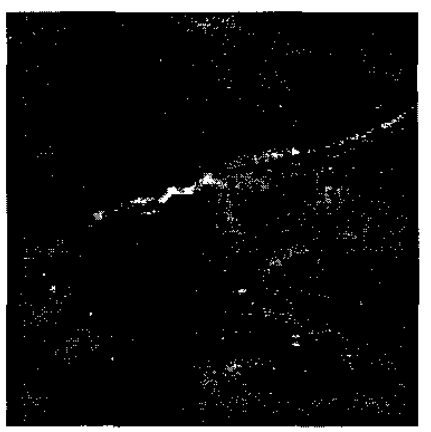

Fig. 11 Fusarium on Two-Photon

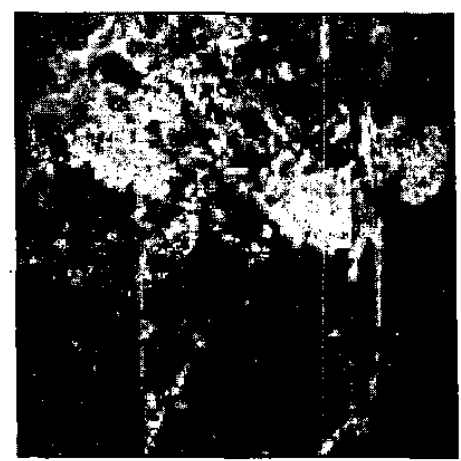

Fig. 9 Asp-flavus on Two-Photon

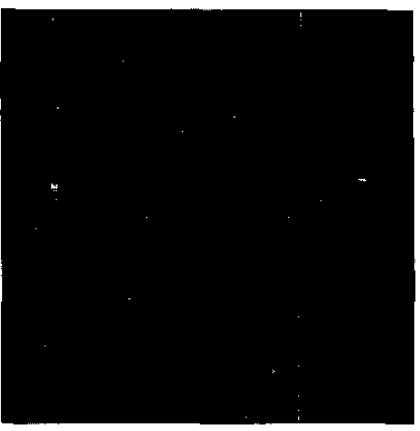

Fig. 12Fusarium on Two-Photon

\section{Conclusion}

We have shown that multiphoton microscopy is a useful imaging modality in the identification of infectious fungi. The primiary luminescence from the fungi is blue and green and their microscopic images and spectroscopic information may be useful in diagnosing fungi infection and developing efficient strategies for their treatment.

\section{$\underline{\text { Reference }}$}

[1] Richard Summerbell Identifying Filamentous Fungi, Guy St-Germain, B Fig. 1, 4, 7, 10 from Identifying Filamentous Fungi 